\title{
Methylene Blue Adsorption by Plasma Re-Activated Carbon
}

\author{
Madeline A. Mackinder1', Keliang Wang1,2, Qi Hua Fan1,2* \\ ${ }^{1}$ Department of Chemical Engineering and Material Science, Michigan State University, East Lansing, MI, USA \\ ${ }^{2}$ Department of Electrical Engineering \& Computer Engineering, Michigan State University, East Lansing, MI, USA \\ Email: *qfan@egr.msu.edu
}

How to cite this paper: Mackinder, M.A., Wang, K.L. and Fan, Q.H. (2021) Methylene Blue Adsorption by Plasma Re-Activated Carbon. Journal of Water Resource and Protection, 13, 778-793.

https://doi.org/10.4236/jwarp.2021.1310041

Received: September 8, 2021

Accepted: October 15, 2021

Published: October 18, 2021

Copyright (c) 2021 by author(s) and Scientific Research Publishing Inc. This work is licensed under the Creative Commons Attribution International License (CC BY 4.0).

http://creativecommons.org/licenses/by/4.0/

(c) (i) Open Access

\begin{abstract}
The effects of $\mathrm{O}_{2}$ plasma treatment on the adsorption capacity of activated carbon $(\mathrm{AC})$ were investigated by varying the plasma treatment times. The surface properties of the AC were characterized by transmission electron microscopy (TEM), X-ray photoelectron spectroscopy (XPS), and Zeta potential. The carbon was then applied to remove methylene blue (MB). The adsorption kinetics and isotherm were also studied. Results showed that pseudo-second-order kinetics was the most suitable model for describing the adsorption of MB onto AC. Equilibrium data were well fitted to the Freundlich isotherm model. The highest adsorption capacity resulted from 4 minutes of $\mathrm{O}_{2}$ plasma treatment. The 4-minute plasma treated AC had the best adsorption capacity for $\mathrm{MB}$ at $0.467 \mathrm{mg} / \mathrm{mg}$. This work shows that activation of AC by plasma can open the micropore and increase the effectiveness of chemical removal.
\end{abstract}

\section{Keywords}

Plasma, Activated Carbon, Methylene Blue

\section{Introduction}

Industrial wastewater holds significant concentrations of toxic chemicals, which pose a significant threat to the environment and public health [1] [2] [3]. Many of these pollutants are resistant to biodegradation and are "forever" if not treated. Conventional methods that have proven effective in treating wastewater include chemical oxidation, membrane treatment, photo-degradation, and adsorption [1] [2] [4]. Most of these technologies are only effective for treating specific chemicals because the pollutants vary in structure and composition.

Ion-exchange resins remove chemicals via electrostatic interactions or van der 
Waals forces. These resins are designed to attractions of the opposite charge [5]. This poses a problem when trying to remove chemicals with opposing charges. Reverse osmosis and nanofiltration are used for treating drinking water on large scales but are expensive [6]. The membranes become fouled and deteriorated, which then requires specialized treatment or disposal [6]. Electrochemical methods are based on electrochemical oxidation, which may form a toxic by-product.

Recent investigations show adsorption by activated carbon (AC) to be an attractive method to remove harmful chemicals from wastewater and are costeffective for treating large volumes [7]. AC is a versatile and effective adsorbent due to its high porosity, large surface area, and surface functional groups [2] [8]. The large adsorption capacity is commonly achieved by chemical activation. Conventional chemical activation of carbon is realized by mixing reactive chemicals (e.g. $\mathrm{NaOH}$ ) with raw carbon materials and subsequently baking at temperatures of $500^{\circ} \mathrm{C}-900^{\circ} \mathrm{C}$. This process can effectively create micropores of $<2 \mathrm{~nm}$ size [9], leading to large surface areas over $1000 \mathrm{~m}^{2} / \mathrm{g}$.

Many as-derived activated carbons exhibit an adsorption capacity inferior to that estimated from their surface area. The main reason is some micropores are not accessible due to impurities. A common practice is to integrate a hightemperature activation stage in combination with the use of excessive amounts of reactive chemicals to create mesopores that open the micropores. Plasma processing produces energetic electrons, ions, and radicals that provide intensive localized heating [10]. Hence, the active species in plasma can improve pore structure at low temperatures. Plasma also offers the ability to generate specific chemical species and functional surface groups through the use of appropriate plasma gases, which allows the AC to be tailored for specific pollutants.

This study examined the adsorption capacity of plasma-treated AC, which was originally activated through conventional chemical activation. Due to its reactivity and etching capabilities, oxygen plasma was studied. The treated AC samples were compared based on adsorption capacity. Plasma treatment offers an alternative to conventional lengthy, and high-temperature activation processes. This work aims to utilize plasma to effectively create mesopores and enhance the adsorption capacity. The material morphology and chemical structure characterization was performed to understand the differences in the adsorption capacity.

\section{Experimental Methods}

\subsection{Plasma System and Process}

A commercial activated carbon (AC R003) received from Oxbow Activated Carbon was used in this study. This chemically activated carbon was further treated through plasma generated by a capacitively coupled dielectric barrier discharge. A quartz tube connected to a mechanical pump was used as a vacuum chamber. A pair of copper electrodes were attached to the outside of the tube to generate plasma. One of the electrodes was connected to a radio frequency (RF) 
power generator (Kurt J. Lesker, Radio Frequency Power Supply R301) while the other electrode was grounded. The operation frequency was set at $13.56 \mathrm{MHz}$. Before the plasma was generated, the quartz tube was pumped down to $<1 \times 10^{-2}$ Torr and purged with the process gas to prevent cross-contamination.

\subsection{Plasma Activation Process}

For plasma activation of the carbon, $\sim 0.05 \mathrm{~g}$ of carbon powder was thinly spread across a ceramic plate. The ceramic plate was placed inside the tube between the two copper electrodes. The system was sealed and pumped down to low pressure. The tube was then filled with the process gas. The plasma pressure was kept constant for each treatment at 2 Torr (measured by a vacuum meter, Kurt J. Lesker). The RF power of the plasma was fixed at $75 \mathrm{~W}$ and adjusted to reach a zero-watt reflection power through all experiments. This process was repeated $5 \mathrm{x}$ for each test in order to reach a final mass of $0.250 \mathrm{~g}$ of treated AC. The plasma gas used was $10 \% \mathrm{O}_{2}-90 \% \mathrm{Ar}$ mixture and treatment times were varied. The plasma treatment times for each gas were: $1 \mathrm{~min}, 2 \mathrm{~min}, 3 \mathrm{~min}, 4 \mathrm{~min}, 5 \mathrm{~min}$, and $6 \mathrm{~min}$.

\subsection{Methylene Blue Batch Experiment}

Methylene blue (MB) was purchased from Acros Organics. A calibration curve was created by measuring the absorbance of differing concentrations of MB solution using a UV-visible spectrophotometer at a wavelength of $664 \mathrm{~nm}$.

The UV-visible spectra of different methylene blue concentrations were used to construct a calibration curve of absorbance versus methylene blue concentration $(\mathrm{mg} / \mathrm{L})$. To do this, samples of methylene blue and DI water with known concentrations were measured by UV-visible spectroscopy (UV-vis). The absorbance peak height for the methylene blue identifying wavelength at $664 \mathrm{~nm}$ was found using Origin. The found peak heights were then plotted against concentration, and a line of best fit was generated in the software. The equation from the line of best fit was then used to calculate final methylene blue concentrations in the following experiments.

Batch adsorption tests were performed at room temperature. The experiments were used to examine the effects of plasma treatment parameters on MB adsorption by AC. The experiments were conducted on samples of AC treated with $\mathrm{O} 2$ plasma and different treatment times (1 - $20 \mathrm{~min}$ ).

To measure the adsorption capacities of each AC sample, $5 \mathrm{mg}$ of AC was added to a glass beaker containing $50 \mathrm{~mL}$ of $10 \mathrm{mg} / \mathrm{L} \mathrm{MB}$ solution. The beaker with the $\mathrm{AC}$ and $\mathrm{MB}$ solution was then placed on a stir plate at room temperature and stirred at $180 \mathrm{rpm}$ for 2 minutes. The solution with the AC was then transferred into a $50 \mathrm{~mL}$ centrifuge tube and was centrifuged at $6000 \mathrm{rpm}$ for 10 minutes to get a clear liquid. The supernatant was then collected and placed in a glass vial. This procedure was repeated for each AC sample.

Once the supernatant from all samples was collected, the remaining concentration of $\mathrm{MB}$ in the solution was measured at $664 \mathrm{~nm}$ using a UV-visible spec- 
trophotometer with a range of $325 \mathrm{~nm}$ to $700 \mathrm{~nm}$. The adsorption capacities of $\mathrm{MB}$ on the AC samples, qt $(\mathrm{mg} / \mathrm{mg})$, were calculated using the following equation:

$$
q_{t}=\frac{V\left(C_{0}-C_{t}\right)}{w}
$$

where $C_{0}(\mathrm{mg} / \mathrm{L})$ is the liquid-phase initial concentration of methylene blue, and $C_{t}(\mathrm{mg} / \mathrm{L})$ is the concentration of methylene blue at each time. The mass of dry AC is represented by $w(\mathrm{~g})$, and $V(\mathrm{~L})$ is the volume of methylene blue solution.

In each experiment, $5 \mathrm{mg}$ of AC was placed in a $50 \mathrm{~mL}$ centrifuge tube. Then $20 \mathrm{~mL}$ of $25 \mathrm{mg} / \mathrm{L} \mathrm{MB}$ solution was added to the centrifuge tube. Then, the centrifuge tubes were spun in the centrifuge at $6000 \mathrm{rpm}$ for 20 minutes. This allowed for the supernatant to separate from the absorbent. The supernatant was then collected and measured for the remaining MB concentration. The remaining concentration was found by measuring the absorbance of the solution by UV-visible spectroscopy at a wavelength of $664 \mathrm{~nm}$. The percent adsorbed and adsorption capacities were calculated using the following equations:

$$
R=\frac{C_{0}-C_{t}}{C_{0}} \times 100
$$

where $R$ is the percentage of $\mathrm{MB}$ adsorbed, $C_{0}(\mathrm{mg} / \mathrm{L})$ is the liquid-phase initial concentration of methylene blue, and $C_{t}(\mathrm{mg} / \mathrm{L})$ is the concentration of methylene blue at each time.

Next, the amount of methylene blue adsorbed by $1.6 \mathrm{~g}$ of $\mathrm{AC}$ at different time increments was investigated. The AC used was not plasma treated and was left in the $36 \mathrm{mg} / \mathrm{L}$ methylene blue solution for the time increments: $0,5,10,20$, and 30 minutes. After the appropriate amount of time, the AC was vacuum filtered from the solution, and this solution without $\mathrm{AC}$ was measured to find the concentration of methylene blue remaining. The final concentration of methylene blue in the solution was measured by UV-vis.

\subsection{Adsorption Kinetics}

Following the same conditions, $50 \mathrm{~mL}$ of $\mathrm{MB}$ solution with the initial concentration of $25 \mathrm{mg} / \mathrm{L}$ was placed in a glass beaker. The solution was left at room temperature and was stirred at $180 \mathrm{rpm}$. Samples were collected at different time intervals and analyzed by UV-visible spectroscopy at the wavelength of $664 \mathrm{~nm}$. The amount of $\mathrm{MB}$ adsorbed onto the $\mathrm{AC}$ was calculated and different kinetic models were applied to fit the process.

\subsection{Adsorption Isotherm}

Following the previous conditions, $20 \mathrm{~mL}$ of $\mathrm{MB}$ solution with different initial concentrations $(5 \mathrm{mg} / \mathrm{L}, 10 \mathrm{mg} / \mathrm{L}, 15 \mathrm{mg} / \mathrm{L}$, and $25 \mathrm{mg} / \mathrm{L})$ was placed in $50 \mathrm{~mL}$ centrifuge tubes. A specific mass of AC of $5 \mathrm{mg}$ was added to the solution in the centrifuge tube and the mixture was spun for an hour at $6000 \mathrm{rpm}$ and then left to 
settle for $24 \mathrm{hrs}$. The final concentration of MB was calculated using UV-visible spectroscopy and different isotherm models were applied to fit the process.

\subsection{Transmission Electron Microscopy}

Plasma treatment was expected to open up the original micropores and create mesopores, which had pore sizes ranging from a few nanometers to about 50 $\mathrm{nm}$. High-magnification microscopy was required to examine porous structures of such scale. Therefore, transmissions electron microscopy (TEM) images were taken of the selected samples using a JEM-2200FS (JEOL, Japan).

\subsection{Zeta Potential}

Samples prepared with a ratio of $1 \mathrm{mg}$ AC to $1 \mathrm{ml}$ DI water were used for the zeta potential measurements. Before the measurement, the samples were ultrasonicated for 20 minutes. The zeta potential measurements were performed on activated carbon using a Zeta potential meter (Malvern Zetasizer Nano-ZS). Each sample was measured three times to provide an average reading.

\subsection{X-Ray Photoelectron Spectroscopy}

Surface analysis by $\mathrm{x}$-ray photoelectron spectroscopy (XPS) was carried out using an SSX-100 system (Surface Science Laboratories, Inc.). XPS was used to analyze surface element composition. High-resolution analysis was performed for $\mathrm{C}, \mathrm{O}$, and $\mathrm{N}$.

\section{Results and Discussion}

\subsection{UV-Visible Spectroscopy of MB Adsorption}

The contact time between $\mathrm{MB}$ and $\mathrm{AC}$ was evaluated as a factor of adsorption efficiency. The UV-vis spectra for $\mathrm{MB}$ adsorption by $\mathrm{AC}$ from 5 to 30 minutes were collected. The results showed that the concentration of MB left in the solution reached a minimum between 10 and 20 minutes (shown in Figure 1), suggesting that the AC became saturated around this time. The concentration decreased $89 \%$ after incubating $\mathrm{MB}$ with $\mathrm{AC}$ for 20 and 30 minutes. The adsorption capacity at equilibrium of the AC before plasma activation was $4.13 \mathrm{mg} \cdot \mathrm{g}^{-1}$.

Next, the effect of different $\mathrm{O}_{2}$ plasma treatment times was investigated. The adsorption capacity was found similar for a plasma treatment time in the range of 3 - 6 minutes but dramatically decreased for treatments longer than $10 \mathrm{mi}-$ nutes, as shown in Figure 2. This could be due to over etching of the AC causing the internal structure to collapse. All further studies were limited to a maximum plasma treatment of 6 minutes in order to avoid over etching.

The effects on adsorption capacity with different plasma treatment times were investigated and compared to non-plasma treated AC. The adsorption capacities of the plasma treated samples significantly increased as shown in Figure 2. The non-plasma treated sample had an adsorption capacity of $4.00 \times 10^{-3} \mathrm{mg} \cdot \mathrm{mg}^{-1}$. As the $\mathrm{O}_{2}$ plasma treatment was applied, the adsorption capacity of the samples 


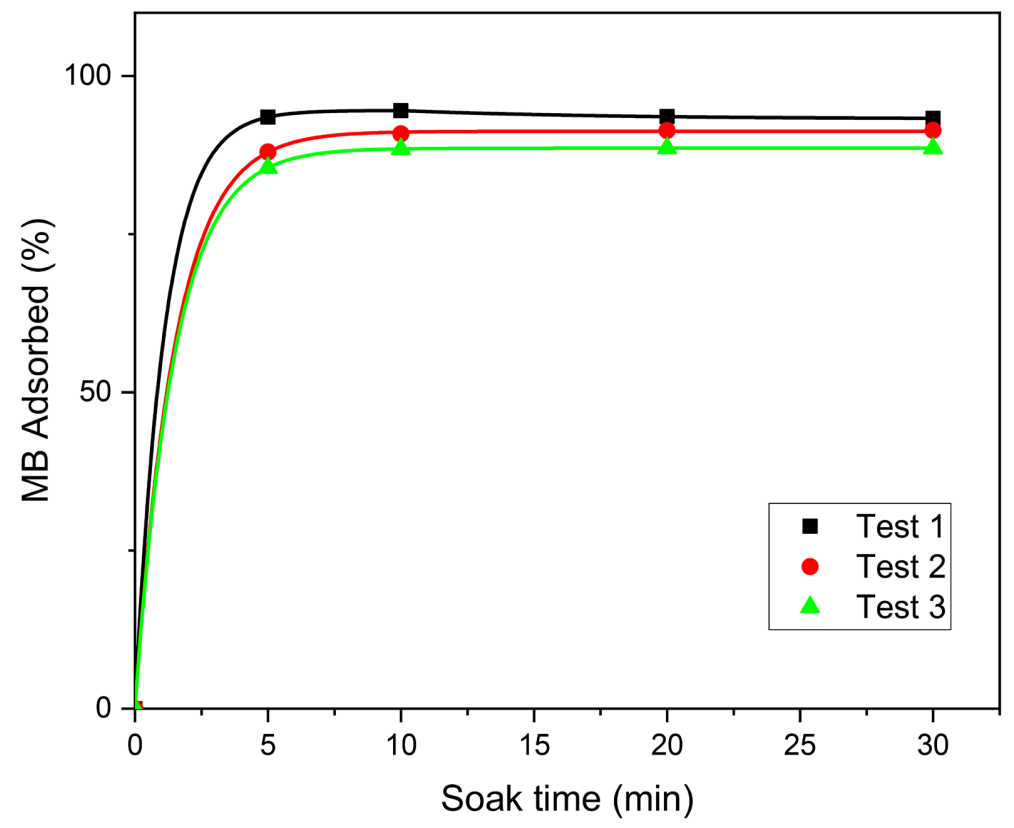

Figure 1. The percent of $\mathrm{MB}$ adsorbed by $\mathrm{AC}$ versus the amount of time that the $\mathrm{AC}$ was soaked in the solution.

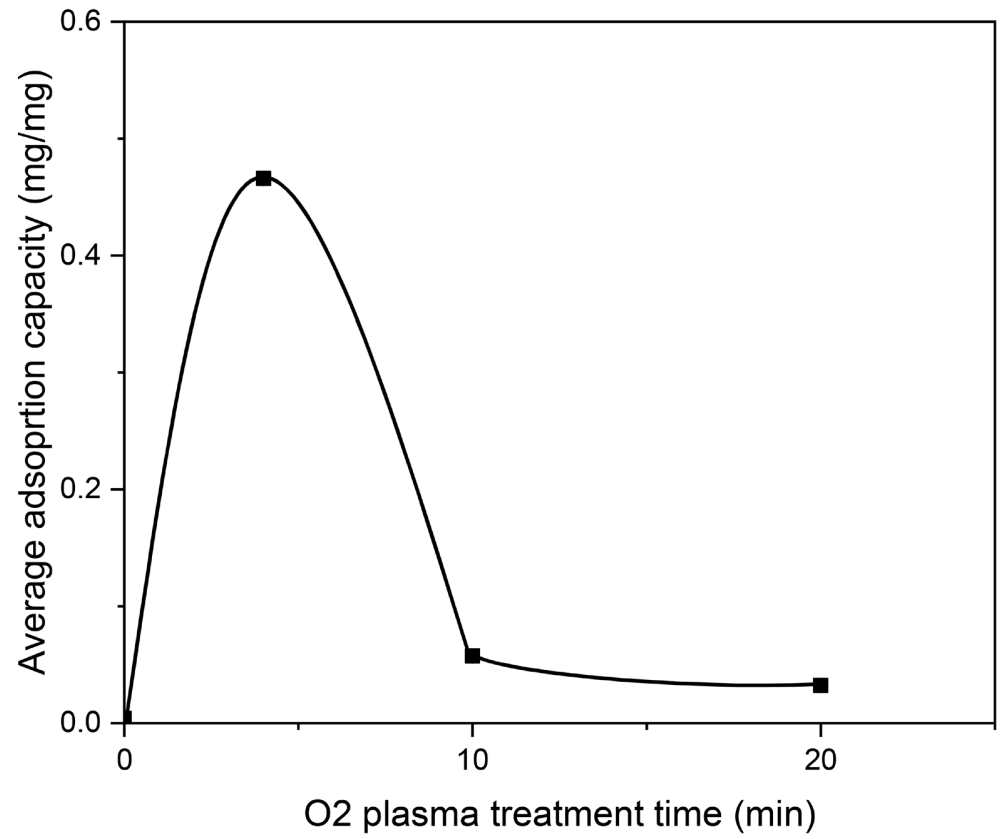

Figure 2. Average adsorption capacity of $\mathrm{O} 2$ plasma treated samples with increasing treatment time.

increased up to 4 minutes. The adsorption capacity for the $\mathrm{O}_{2}$ plasma treated samples reached its maximum adsorption capacity of $0.467 \mathrm{mg} \cdot \mathrm{mg}^{-1}$ at $4 \mathrm{mi}$ nutes of plasma treatment. After 4 minutes of treatment, the adsorption capacity decreased. After 10 minutes of $\mathrm{O}_{2}$ plasma treatment, the adsorption capacity had decreased $\sim 88 \%$ to $0.057 \mathrm{mg} / \mathrm{mg}$, and after 20 minutes it had decreased $\sim 93 \%$ to $0.032 \mathrm{mg} / \mathrm{mg}$. Overall, the $\mathrm{O}_{2}$ plasma treated samples had adsorption capacities 
higher than the non-plasma treated sample. The best adsorption capacity for the $\mathrm{O}_{2}$ plasma treatment was found to be $0.467 \mathrm{mg} \cdot \mathrm{mg}^{-1}$ at 4 minutes of plasma treatment which was over a one hundred times increase. The significant increase showed that plasma treatment of AC increases adsorption capacity.

A simple experiment was conducted to ensure that the increase in adsorption capacity was not due to the removal of residual water from vacuum and localized heating. The adsorption capacity of AC left in vacuum for 10 minutes and the adsorption capacity of $\mathrm{AC}$ left in $110^{\circ} \mathrm{C}$ oven for 10 minutes were compared to untreated AC (Figure 3). Figure 4 shows that the influence of heat and vacuum

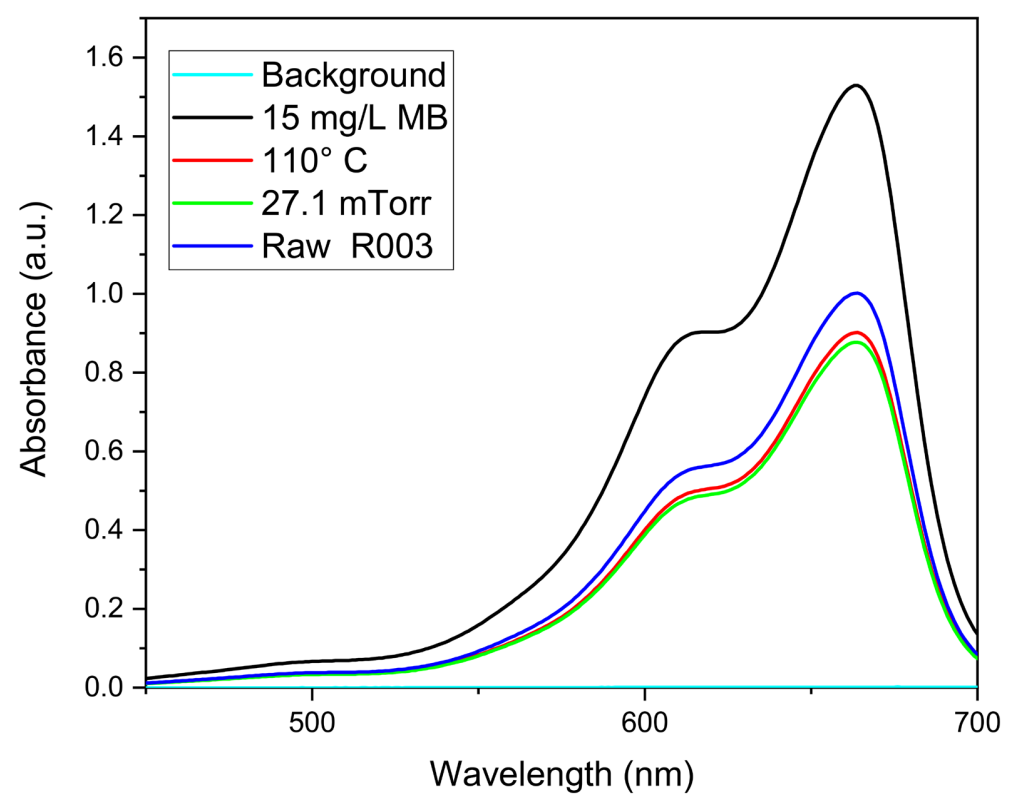

Figure 3. MB adsorption of AC that was heated, left in vacuum, or raw.

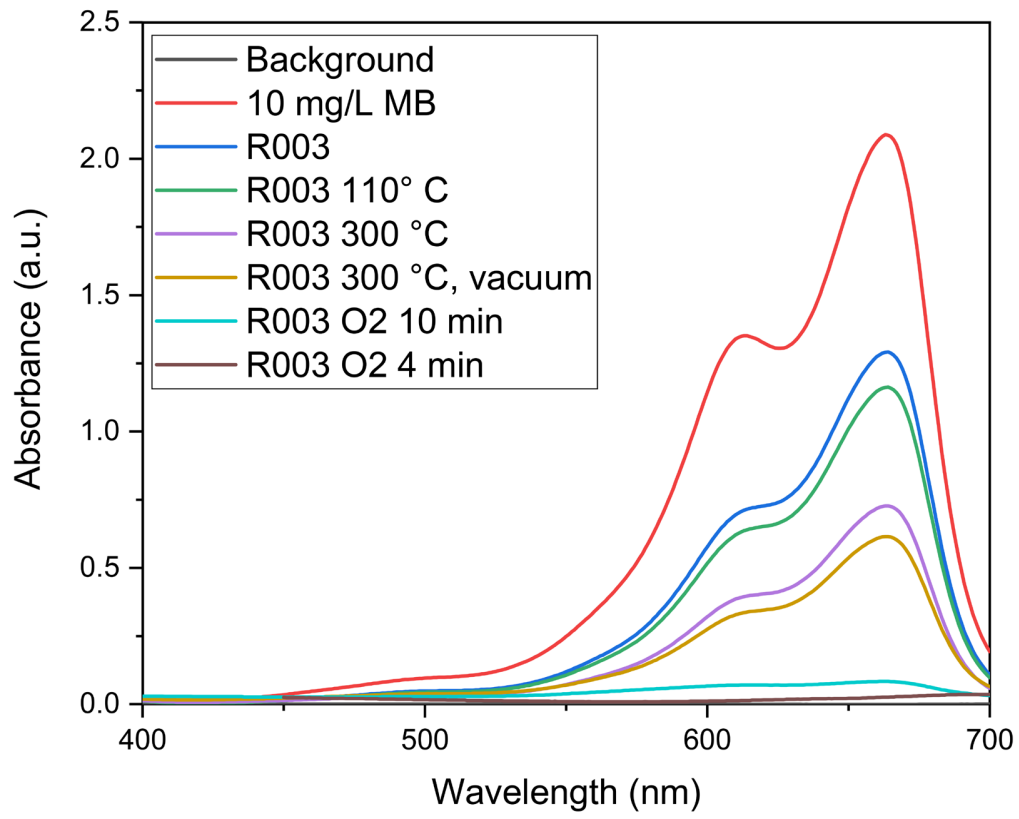

Figure 4. $\mathrm{MB}$ adsorption of $\mathrm{AC}$ under various experimental conditions. 
on the adsorption capacity of MB by the AC is insignificant.

To better explain how all the plasma treatments led to similar adsorption capacities, the original AC R003 was further examined. The AC was heated to $110^{\circ} \mathrm{C}-300^{\circ} \mathrm{C}$ for 30 minutes with and without being kept under vacuum. The treatments included in Figure 4 show that an increased temperature in concurrence with treatment under vacuum improved $\mathrm{MB}$ adsorption for the nonplasma treated samples. Then, the effect of treating $\mathrm{AC}$ with $\mathrm{O}_{2}$ plasma at room temperature was compared with the non-plasma treated samples. The graph clearly shows that the 4-minute $\mathrm{O}_{2}$ plasma treated sample had the highest adsorption capacity. The overall results suggest that the plasma treatment provided significant localized heating at atomic scale in addition to modifying the porous structure.

\subsection{Transmission Electron Microscopy}

Transmission electron microscopy (TEM) is used to obtain information about the morphology and microstructure of materials [11] [12]. TEM transmits a high energy electron beam through a sample to form an image. TEM images were taken of the best performing samples from the plasma treatment, along with the untreated AC.

Figure 5 shows TEM images of non-plasma treated AC (left) and $\mathrm{O}_{2}$ plasma treated AC (right). Brighter areas in the images indicate that more electrons were able to penetrate the sample. Hence, the brighter areas are pores. The scale bars for each image differ due to the need for higher magnification to see the porous structure in the non-plasma treated sample. The image shows that the non-plasma treated AC contains mainly micropores. With plasma treatment, meso-pores with irregular shapes appear because the AC is etched by the plasma. The larger mesopores are easily visible under lower magnification. This combined meso-pores and micro-pores facilitate the transport of $\mathrm{MB}$ and promote the adsorption.
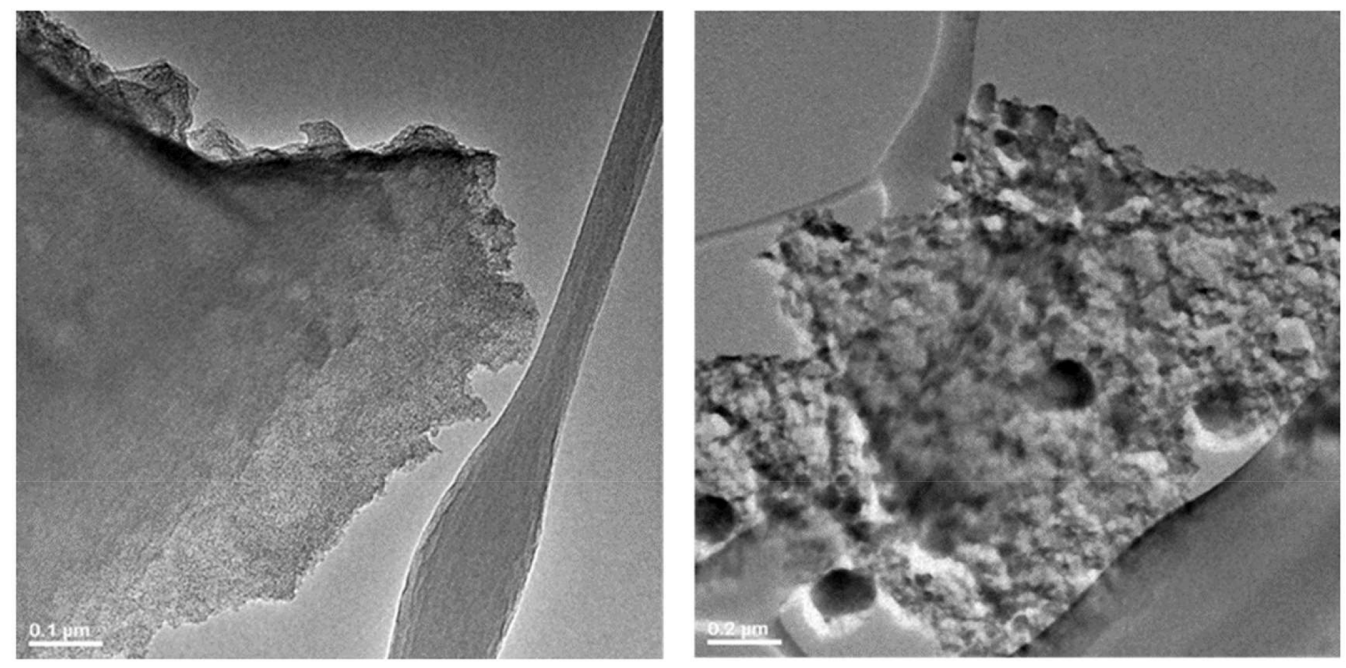

Figure 5. TEM images of non-plasma treated AC (left) and $\mathrm{O}_{2}$ plasma treated AC (right). 


\subsection{XPS}

The XPS analysis of AC is shown in Figure 6. XPS provides information about the surface functional species and composition. Carbon, oxygen, and nitrogen content were determined. The XPS analysis of AC that was not plasma treated gave the atomic concentrations $89.01 \% \mathrm{C} 1$ s and $10.99 \% \mathrm{O} 1 \mathrm{~s}$. No $\mathrm{N}$ 1s was detected. The high-resolution spectrum of C 1s of untreated AC could be deconvoluted into 3 peaks: 284.74, 286.35, and $287.90 \mathrm{eV}$. The $\mathrm{O} 1 \mathrm{~s}$ region was made up of three peaks: 529.5, 531.1, and $532.5 \mathrm{eV}$.

The XPS analysis of AC that was treated with $\mathrm{O}_{2}$ plasma for 4 minutes gave the atomic concentrations $80.06 \% \mathrm{C}$ 1s and $19.94 \% \mathrm{O} 1 \mathrm{~s}$ with no apparent $\mathrm{N}$. The high-resolution spectrum of $\mathrm{C} 1 \mathrm{~s}$ of $\mathrm{AC}$ that underwent $\mathrm{O}_{2}$ plasma treatment for 4 minutes can be broken into 5 peaks: 284.82, 285.42, 286.37, 287.92,
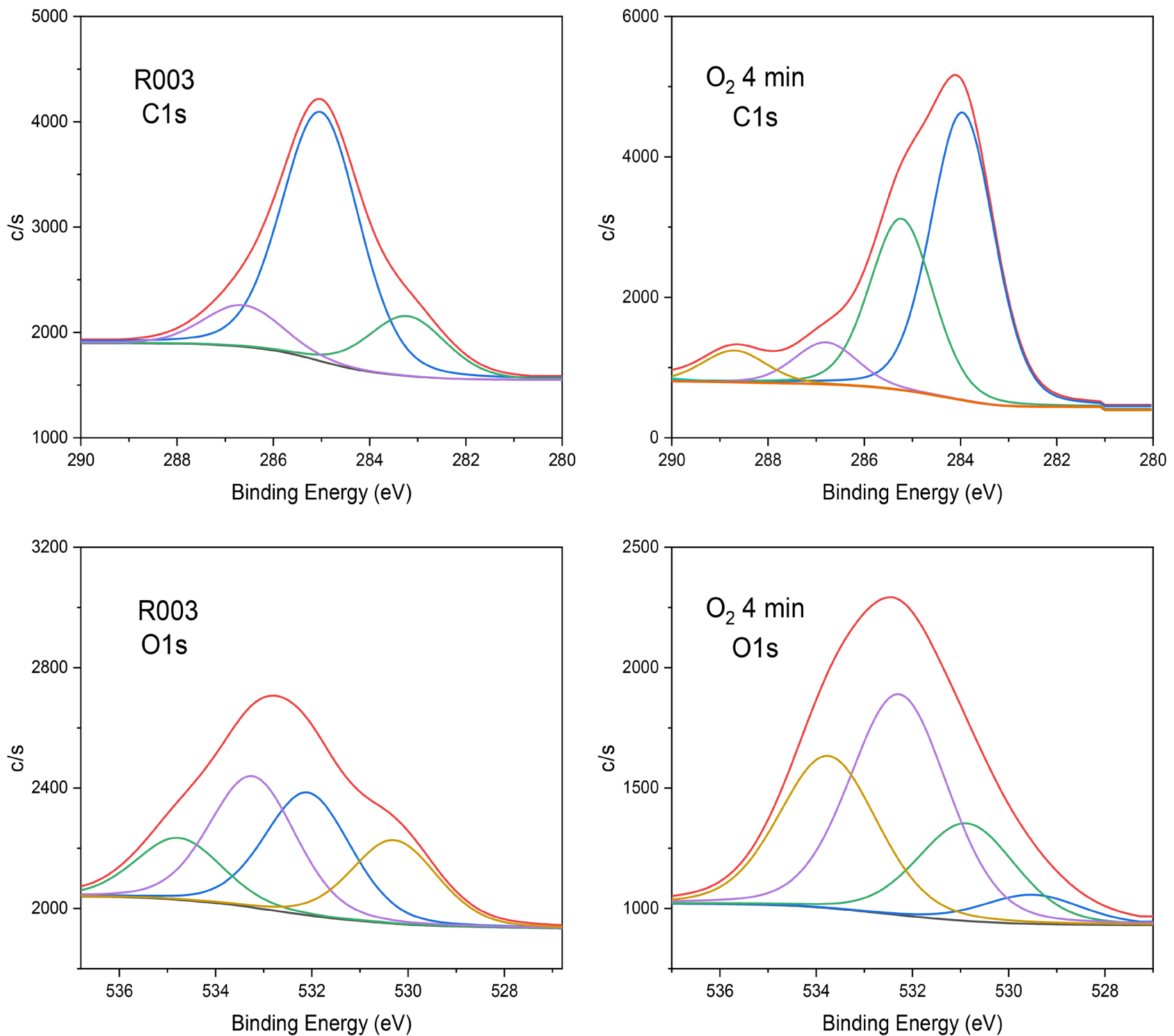

Figure 6. XPS data for non-plasma treated AC (top and bottom left) and AC that underwent $\mathrm{O}_{2}$ plasma treatment for 4 minutes (top and bottom right). 
and $289.37 \mathrm{eV}$. The $\mathrm{O} 1 \mathrm{~s}$ region was split into 2 peaks: 533.88 and $532.63 \mathrm{eV}$.

Compared to the untreated AC, XPS analysis showed a decrease in carbon concentration in the $\mathrm{O}_{2}$ plasma treated sample. There was no discernable nitrogen found in the $\mathrm{O}_{2}$ plasma treated sample, but there was an increase in oxygen. The increase in oxygen with a decrease in carbon indicates a change in polarity [4]. The samples with higher amounts of oxygen would be expected to be more polar and have less aromaticity leading to less hydrophobicity [4]. There was no discernable nitrogen in the $\mathrm{O}_{2}$ sample or the non-plasma treated sample. XPS analysis further revealed a change in surface functional groups and atomic composition after plasma treatment.

The peak numbers and their interpretation are listed in Table 1. With plasma treatment, the C1s XPS spectra could be broken down into more peaks. Plasma treatment of $\mathrm{AC}$ resulted in an increase in $\mathrm{C}-\mathrm{O}-\mathrm{C}$ and $\mathrm{C}=\mathrm{O}$ functional groups. In the $\mathrm{O}_{2}$ plasma treated sample, a peak around $290 \mathrm{eV}$ appeared. This peak corresponds to $\mathrm{C}=\mathrm{O} / \mathrm{C}=\mathrm{C}$ (carbonate, occluded $\mathrm{CO}, \pi$-electrons in aromatic rings) [9] [13] [14] [15].

With plasma treatment the O1s XPS spectra of the $\mathrm{O}_{2}$ sample only had two peaks. The $\mathrm{O}_{2}$ treated sample loses its $\mathrm{O}$ (oxide-like oxygen) and its $\mathrm{C}=\mathrm{O}$ (quinone type carbonyl) peaks, and it gains a peak at $533.9 \mathrm{eV}$ that corresponds with C-O (ester/amide/carboxylic anhydride) [13] [16] [17] [18] [19].

\subsection{Zeta Potential}

Zeta potential measurements were performed on the $\mathrm{O}_{2}$ and the non-plasma treated AC sample. Figure 7 shows the comparison between each sample when the adsorption capacity was the highest. The $\mathrm{O}_{2}$ plasma-treated AC has the most negative zeta potential compared to the non-plasma treated AC sample. The zeta potential curve for $\mathrm{O}_{2}$ plasma-treated $\mathrm{AC}$ shifts to the left of the non-plasma treated AC. The zeta potential of the $\mathrm{O}_{2}$ plasma-treated AC is measured at -63 $\mathrm{mV}$.

Table 1. The assignment of the XPS peaks.

\begin{tabular}{|c|c|c|c|}
\hline Peak (eV) & Assignment & Surface group & Reference \\
\hline \multicolumn{4}{|l|}{$O 1 s$} \\
\hline $532.9-533.2 \mathrm{eV}$ & $\begin{array}{l}\text { Ester/amide/carboxylic anhydrides and } \\
\text { oxygen atoms in hydroxyls or ethers }\end{array}$ & $\mathrm{C}-\mathrm{O}$ & [13] [16] [17] [18] [19] \\
\hline $534.2 \mathrm{eV}$ & Carboxylic acid & $\mathrm{C}-\mathrm{O}$ & [13] [16] [17] [18] [19] \\
\hline \multicolumn{4}{|l|}{$C 1 s$} \\
\hline $284.2-284.9 \mathrm{eV}$ & Graphite & $\mathrm{C}$ & [9] [13] [14] [15] \\
\hline $285.4-286.3 \mathrm{eV}$ & Ether/phenol/alcohol & $\mathrm{C}-\mathrm{O}-$ & [9] [13] [14] [15] \\
\hline $287.2-287.9 \mathrm{eV}$ & Carbonyl/quinone & $\mathrm{C}=\mathrm{O}$ & [9] [13] [14] [15] \\
\hline $288.7-289.3 \mathrm{eV}$ & Carboxyl/ester & $\mathrm{COO}$ & [9] [13] [14] [15] \\
\hline $290.2-290.8 \mathrm{eV}$ & $\begin{array}{l}\text { Carbonate, occluded CO, } \\
\pi \text {-electrons in aromatic ring }\end{array}$ & $\mathrm{C}=\mathrm{O} / \mathrm{C}=\mathrm{C}$ & [9] [13] [14] [15] \\
\hline
\end{tabular}




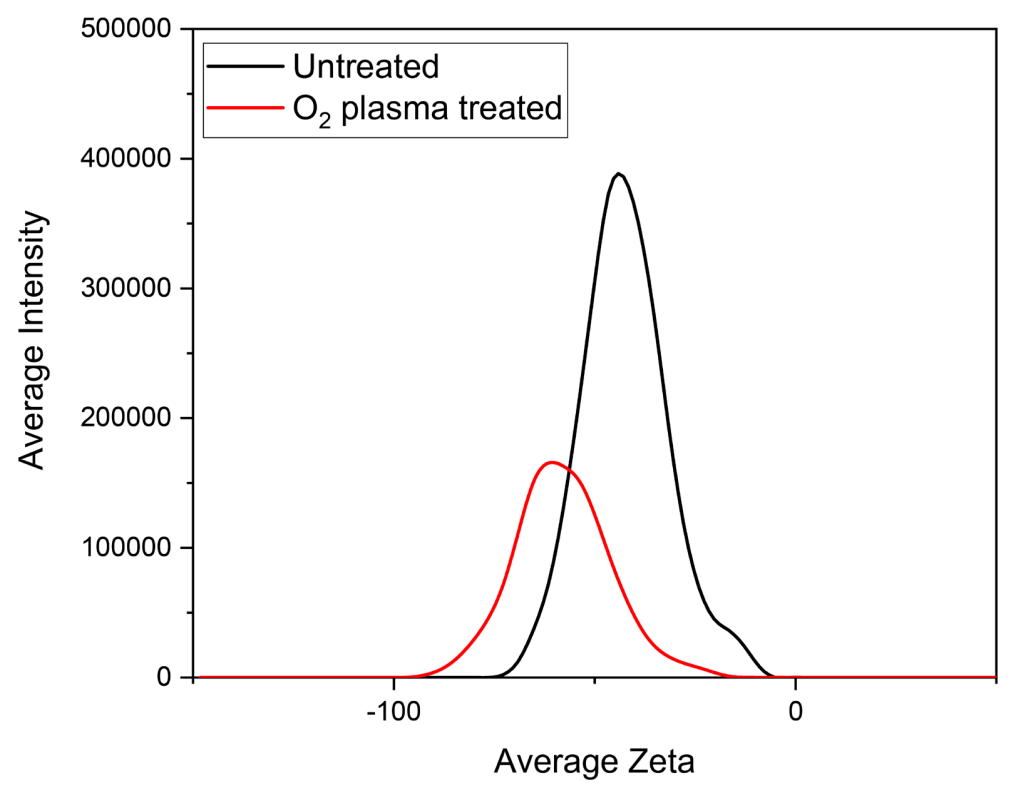

Figure 7. Zeta potential data of non-plasma and $\mathrm{O}_{2}$ plasma treated AC samples.

Zeta potential is a measure of surface charge. It is the potential between the dispersed particle surface and the mobile dispersion medium [20]. It can be observed in Figure 7 that all the adsorbents had large negative zeta potentials. This implies that the particles in suspension resist aggregation and are inclined to disperse homogeneously throughout the solution [20]. This opposition to aggregation and the ability to spread evenly throughout a solution is beneficial to adsorption. The magnitude of the zeta potential is indicative of colloidal stability [21]. Figure 7 clearly shows that the $\mathrm{O}_{2}$ plasma treated sample has a zeta potential between $-35 \mathrm{mV}$ and $-65 \mathrm{mV}$. Nanoparticles with zeta potentials less than $-30 \mathrm{mV}$ are considered to have high degrees of stability [21].

\subsection{Kinetics of Adsorption}

Adsorption of dyes on AC may include chemical adsorption. To investigate the adsorption mechanism, the adsorption kinetics of $\mathrm{MB}$ onto $\mathrm{AC}$ that was not plasma treated was analyzed by using pseudo-first and pseudo-second order kinetic models. The pseudo-first-order equation can be written in the form:

$$
\log \left(q_{e}-q_{t}\right)=\log \left(q_{e}\right)-\frac{k_{1}}{2.303} t
$$

where $q_{e}$ represents the amount of adsorption $(\mathrm{mg} / \mathrm{mg})$ at equilibrium, $k_{1}$ is the rate constant of the pseudo-first-order equation, and $q_{t}$ is the amount of adsorption $(\mathrm{mg} / \mathrm{mg})$ at $t$, time $(\mathrm{min})$ [22]. The rate constant, $k_{1}$, can be calculated by plotting $\log \left(q_{e}-q_{t}\right)$ versus $t[22]$.

The pseudo-second-order kinetic equation is shown below:

$$
\frac{t}{q_{t}}=\frac{1}{k_{2} q_{e}^{2}}+\frac{1}{q_{e}} t
$$

where $k_{2}$ is the pseudo-second-order rate constant. Plotting $t / q_{t}$ versus $t$, a line of 
best fit can be found (Figure 8). This straight line can then be used to calculate $k_{2}$ [22]. The $R^{2}$ value for the pseud-second-order model was 0.99 , where the $R^{2}$ value for the pseudo-first-order model was only 0.80 . Therefore, the adsorption kinetics are adequately described by the pseudo-second-order model. Similar results have been found for the adsorption of $\mathrm{MB}$ onto rejected tea leaves, $\mathrm{AC}$, and different carbonaceous materials [20] [22] [23].

The pseudo-second order model fits the kinetics data of $\mathrm{MB}$ adsorption onto AC. This suggests that chemisorption has a dominant role in the adsorption process [16] [23].

\subsection{Adsorption Isotherms}

The relationship between the initial concentration of $\mathrm{MB}$ and the adsorbed amount of $\mathrm{MB}$ onto $\mathrm{AC}$ are investigated and shown in Figure 9. The graphs show that the adsorption of $\mathrm{MB}$ increases with the increase of the equilibrium concentration of MB.

The two isotherms tested for their ability to describe the experimental results were the Langmuir and Freundlich adsorption isotherms [24]. These models provide insight into the mechanism of adsorption and the surface properties [24]. The Langmuir isotherm is based on the assumption of monolayer adsorption onto a homogeneous surface [22] [24]. The Langmuir isotherm is expressed by the following equation:

$$
\frac{C_{e}}{q_{e}}=\frac{1}{q_{\max } b}+\frac{1}{q_{\max }} C_{e}
$$

where $C_{e}$ is the equilibrium concentration $(\mathrm{mg} / \mathrm{L}), q_{e}$ is the adsorption capacity at equilibrium $(\mathrm{mg} / \mathrm{g}), b$ is the Langmuir constant $(\mathrm{L} / \mathrm{g}), q_{\max }$ is the maximum

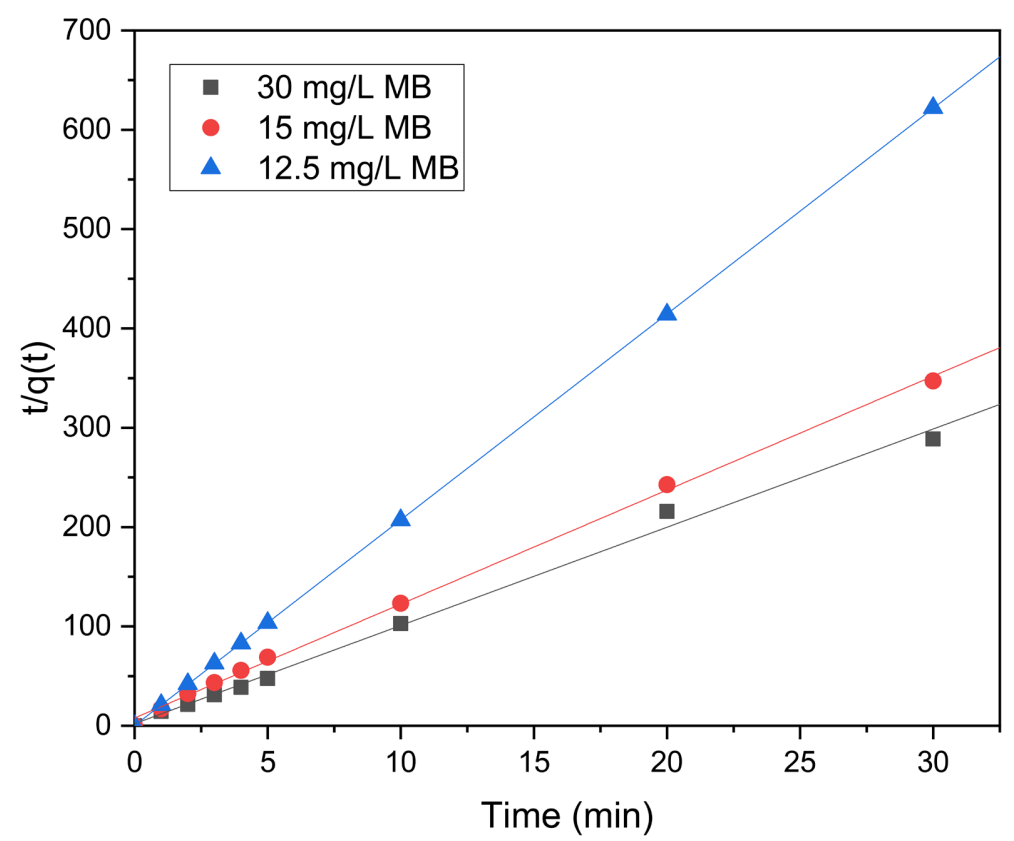

Figure 8. Plot of $t / q_{t}$ versus $t$ showing a line of best fit for the pseud-second-order model. 

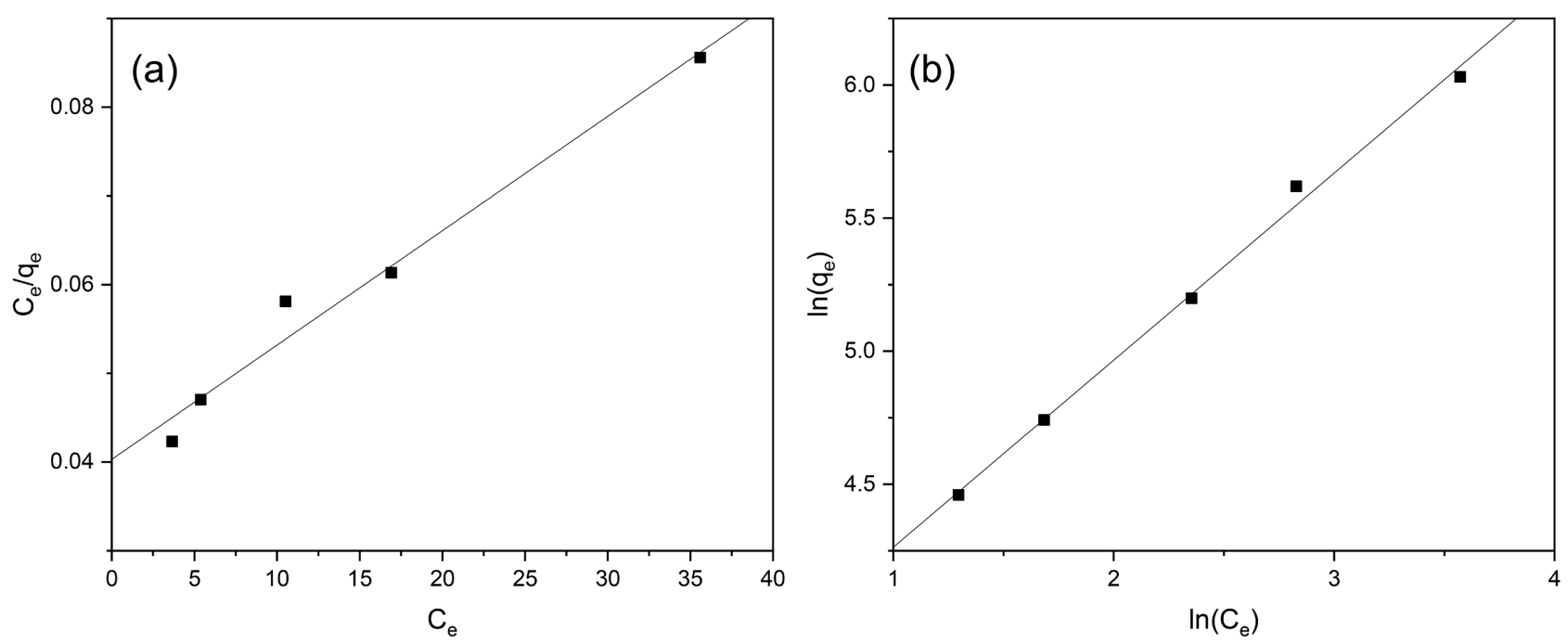

Figure 9. Langmuir (a) and Freundlich (b) isotherm adsorption models.

adsorption capacity (mg/g).

The Freundlich isotherm is related to adsorption capacity and intensity [22]. The Freundlich isotherm assumes a heterogeneous surface with a non-uniform distribution of adsorption [22]. It is expressed by the following equation:

$$
\ln q_{e}=\ln K_{f}+\frac{1}{n} \ln C_{e}
$$

where $C_{e}$ is the equilibrium concentration $(\mathrm{mg} / \mathrm{L}), q_{e}$ is the adsorption capacity at equilibrium (mg/g), $K_{f}$ is the Freundlich constant $(\mathrm{L} / \mathrm{mg}), 1 / n$ is the heterogeneity of the sorption sites and an indicator of isotherm nonlinearity [23].

The data was fit to the Langmuir and Freundlich isotherm adsorption models. The data fitting gave the regression coefficients Freundlich $\left(R^{2}>0.996\right.$ and the Langmuir $\left(R^{2}>0.977\right)$ models indicating that the models described the adsorption process adequately. This indicates that the adsorption of MB onto activated carbon occurred primarily by single molecular adsorption [23].

\section{Conclusion}

As-produced activated carbon (AC) is dominated by micropores and often contains some type of contamination that interferes with the adsorption of MB. This work aims to modulate the microstructure of $\mathrm{AC}$ and eliminate the surface contamination using plasma, which provides localized heating at the atomic scale and enhances the activation process. The results showed that the combination of heat, vacuum, and plasma-treatment could effectively remove the contamination, significantly increasing the adsorption capacity. The maximum adsorption capacity of $\mathrm{MB}$ by $\mathrm{O}_{2}$ plasma treated $\mathrm{AC}$ was $0.467 \mathrm{mg} \cdot \mathrm{mg}^{-1}$ which was significantly higher than that of the untreated AC. The results of XPS analysis indicated a change in surface functional groups and atomic composition after plasma treatment. The surface groups shown in both the $\mathrm{O}_{2}$ plasma treated and non-plasma treated samples mainly consisted of carboxylic groups, phenols 
and/or ethers, and carbonyls. After plasma treatment $\mathrm{C}-\mathrm{O}-\mathrm{C}$ and $\mathrm{C}=\mathrm{O}$ functional groups appeared. The adsorption kinetics and isotherm were also studied. Results showed that pseudo-second-order kinetics was the most suitable model for describing the adsorption of $\mathrm{MB}$ onto AC. Equilibrium data were well fitted to the Freundlich isotherm model.

\section{Acknowledgements}

The authors gratefully acknowledge Zac Seeds and William Lee for their help plasma treating samples. This work was partially supported by the National Science Foundation (Grants No. 1917577, No. 1724941) and by the 21 sty Century Jobs Trust Fund received through the MSU and the State of Michigan. Also acknowledged is Michigan Translational Research and Commercialization program.

\section{Conflicts of Interest}

The authors declare no conflicts of interest regarding the publication of this paper.

\section{References}

[1] Behazin, E., Ogunsona, E., Rodriguez-Uribe, A., Mohanty, A.K., Misra, M. and Anyia, A.O. (2015) Mechanical, Chemical, and Physical Properties of Wood and Perennial Grass Biochars for Possible Composite Application. BioResources, 11, 1334-1348. https://doi.org/10.15376/biores.11.1.1334-1348

[2] Melo, L.C.A., Coscione, A.R., Abreu, C.A., Puga, A.P. and Camargo, O.A. (2013) Influence of Pyrolysis Temperature on Cadmium and Zinc Sorption Capacity of Sugar Cane Straw-Derived Biochar. BioResources, 8, 4992-5004.

https://doi.org/10.15376/biores.8.4.4992-5004

[3] De Figueredo, N.A., et al. (2017) Characterization of Biochars from Different Sources and Evaluation of Release of Nutrients and Contaminants. Revista Ciência Agronômica, 48, 395-403. https://doi.org/10.5935/1806-6690.20170046

[4] Cantrell, K.B., Hunt, P.G., Uchimiya, M., Novak, J.M. and Ro, K.S. (2012) Impact of Pyrolysis Temperature and Manure Source on Physicochemical Characteristics of Biochar. Bioresource Technology, 107, 419-428.

https://doi.org/10.1016/j.biortech.2011.11.084

[5] Green, D.W. and Perry, R.H. (2008) Perry's Chemical Engineers' Handbook. 8th Edition, McGraw-Hill Education, New York.

[6] Wimalawansa, S.J. (2021) Purification of Contaminated Water with Reverse Osmosis: Effective Solution of Providing Clean Water for Human Needs in Developing Countries. International Journal of Emerging Technology and Advanced Engineering, 3, 75-89.

[7] Angin, D., Altintig, E. and Köse, T.E. (2013) Influence of Process Parameters on the Surface and Chemical Properties of Activated Carbon Obtained from Biochar by Chemical Activation. Bioresource Technology, 148, 542-549.

https://doi.org/10.1016/j.biortech.2013.08.164

[8] Hao, F., et al. (2013) Molecular Structure of Corncob-Derived Biochars and the Mechanism of Atrazine Sorption. Agronomy Journal, 105, 773-782. 
https://doi.org/10.2134/agronj2012.0311

[9] Liu, Q.S., Zheng, T., Wang, P. and Guo, L. (2010) Preparation and Characterization of Activated Carbon from Bamboo by Microwave-Induced Phosphoric Acid Activation. Industrial Crops and Products, 31, 233-238. https://doi.org/10.1016/j.indcrop.2009.10.011

[10] Konno, K., et al. (2017) Short-Time Preparation of NaOH-Activated Carbon from Sugar Cane Bagasse Using Microwave Plasma Heating. Green and Sustainable Chemistry, 7, 259-269. https://doi.org/10.4236/gsc.2017.74020

[11] Somani, P.R. and Umeno, M. (2007) Importance of Transmission Electron Microscopy for Carbon Nanomaterials Research. In: Méndez-Vilas, A. and Díaz, J., Eds., Modern Research and Educational Topics in Microscopy, FORMATEX, 634-642. https://www.researchgate.net/profile/Masayoshi_Umeno/publication/228758446_I mpor-

tance_of_Transmission_Electron_Microscopy_for_Carbon_Nanomaterials_Researc h/links/0c96053bb5dc9d2fb9000000/Importance-of-Transmission-Electron-Micros copy-for-Carbon-Nanomaterials-Research.pdf

[12] Jurkiewicz, K., Pawlyta, M. and Burian, A. (2018) Structure of Carbon Materials Explored by Local Transmission Electron Microscopy and Global Powder Diffraction Probes. Journal of Carbon Research, 4, Article No. 68.

https://doi.org/10.3390/c4040068

[13] Moulder, J.F., Stickle, W.F., Sobol, P.E., Bomben, K.D. and Chastain, J. (1995) Handbook of X-Ray Photoelectron Spectroscopy: A Reference Book of Standard Spectra for Identification and Interpretation of XPS Data. Physical Electronics, Chanhassen.

[14] Terzyk, A.P. (2001) The Influence of Activated Carbon Surface Chemical Composition on the Adsorption of Acetaminophen (Paracetamol) in Vitro. Part II. TG, FTIR, and XPS Analysis of Carbons and the Temperature Dependence of Adsorption Kinetics at the Neutral pH. Colloids and Surfaces A: Physicochemical and Engineering Aspects, 177, 23-45. https://doi.org/10.1016/S0927-7757(00)00594-X

[15] Ryu, Z., Rong, H., Zheng, J., Wang, M. and Zhang, B. (2002) Microstructure and Chemical Analysis of PAN-Based Activated Carbon Fibers Prepared by Different Activation Methods. Carbon, 40, 1144-1147.

https://doi.org/10.1016/S0008-6223(02)00105-7

[16] Liu, L., Fan, S. and Li, Y. (2018) Removal Behavior of Methylene Blue from Aqueous Solution by Tea Waste: Kinetics, Isotherms and Mechanism. International Journal of Environmental Research and Public Health, 15, Article No. 1321. https://doi.org/10.3390/ijerph15071321

[17] Armelao, L., et al. (2003) Introduction to XPS Studies of Metal and Metal-Oxide Nanosystems. Surface Science Spectra, 10, 137-142.

https://doi.org/10.1116/11.20050199

[18] Jones, T.E., Rocha, T.C.R., Knop-Gericke, A., Stampfl, C., Schlögl, R. and Piccinin, S. (2015) Insights into the Electronic Structure of the Oxygen Species Active in Alkene Epoxidation on Silver. ACS Catalysis, 5, 5846-5850. https://doi.org/10.1021/acscatal.5b01543

[19] Lennon, D., Lundie, D.T., Jackson, S.D., Kelly, G.J. and Parker, S.F. (2002) Characterization of Activated Carbon Using X-Ray Photoelectron Spectroscopy and Inelastic Neutron Scattering Spectroscopy. Langmuir, 18, 4667-4673.

https://doi.org/10.1021/la011324j

[20] Li, Y., et al. (2013) Comparative Study of Methylene Blue Dye Adsorption onto Ac- 
tivated Carbon, Graphene Oxide, and Carbon Nanotubes. Chemical Engineering Research and Design, 91, 361-368. https://doi.org/10.1016/j.cherd.2012.07.007

[21] Kumar, A. and Dixit, C.K. (2017) 3-Methods for Characterization of Nanoparticles. In: Nimesh, S., Chandra, R. and Gupta, N., Eds., Advances in Nanomedicine for the Delivery of Therapeutic Nucleic Acids, Woodhead Publishing, Cambridge, 43-58. https://doi.org/10.1016/B978-0-08-100557-6.00003-1

[22] Nasuha, N., Hameed, B.H. and Din, A.T.M. (2010) Rejected Tea as a Potential Low-Cost Adsorbent for the Removal of Methylene Blue. Journal of Hazardous Materials, 175, 126-132. https://doi.org/10.1016/j.jhazmat.2009.09.138

[23] Fan, S., et al. (2016) Biochar Prepared from Co-Pyrolysis of Municipal Sewage Sludge and Tea Waste for the Adsorption of Methylene Blue from Aqueous Solutions: Kinetics, Isotherm, Thermodynamic and Mechanism. Journal of Molecular Liquids, 220, 432-441. https://doi.org/10.1016/j.molliq.2016.04.107

[24] Chen, L., Ramadan, A., Lü, L., Shao, W., Luo, F. and Chen, J. (2011) Biosorption of Methylene Blue from Aqueous Solution Using Lawny Grass Modified with Citric Acid. Journal of Chemical \& Engineering Data, 56, 3392-3399.

https://doi.org/10.1021/je200366n 